\title{
Clinical Experience of 1-Minute Brain MRI Using a Multicontrast EPI Sequence in a Different Scan Environment
}

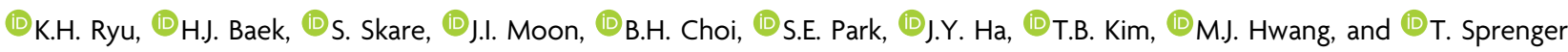
ow

\begin{abstract}
BACKGROUND AND PURPOSE: The long scan time of MR imaging is a major drawback limiting its clinical use in neuroimaging; therefore, we aimed to investigate the clinical feasibility of a 1-minute full-brain MR imaging using a multicontrast EPI sequence on a different MR imaging scanner than the ones previously reported.
\end{abstract}

MATERIALS AND METHODS: We retrospectively reviewed the records of 146 patients who underwent a multicontrast EPI sequence, including T1-FLAIR, T2-FLAIR, T2WI, DWI, and T2*WI sequences. Two attending neuroradiologists assessed the image quality of each sequence to compare the multicontrast EPI sequence with routine MR imaging protocols. We used the Wilcoxon signed rank test and McNemar test to compare the 2 MR imaging protocols.

RESULTS: The multicontrast EPI sequence generally showed sufficient image quality of $>2$ points using a 4-point assessment scale. Regarding image quality and susceptibility artifacts, there was no significant difference between the multicontrast EPI sequence DWI and routine DWI $(P>.05)$, attesting to noninferiority of the multicontrast EPI, whereas there were significant differences in the other 4 sequences between the 2 MR imaging protocols.

CONCLUSIONS: The multicontrast EPI sequence showed sufficient image quality for clinical use with a shorter scan time; however, it was limited by inferior image quality and frequent susceptibility artifacts compared with routine brain MR imaging. Therefore, the multicontrast EPI sequence cannot completely replace the routine MR imaging protocol at present; however, it may be a feasible option in specific clinical situations such as screening, time-critical diseases or for use with patients prone to motion.

ABBREVIATIONS: EPIMix = multicontrast EPI sequence; SWAN = susceptibility-weighted angiography

$\mathbf{T}$ he long scan time of brain MR imaging is a major drawback limiting its clinical use. To shorten the scan time, fast imaging techniques have been introduced. ${ }^{1-6}$ However, most studies have examined specific clinical conditions, thus

Received October 4, 2019; accepted after revision January 2, 2020.

From the Department of Radiology (K.H.R., H.J.B., J.I.M., B.H.C., S.E.P., J.Y.H., T.B.K.), Gyeongsang National University School of Medicine and Gyeongsang National University Changwon Hospital, Changwon, Republic of Korea; Department of Radiology (H.J.B.), Institute of Health Sciences, Gyeongsang National University School of Medicine, Jinju, Republic of Korea; Department of Clinical Neuroscience (S.S., T.S.), Karolinska Institute, Stockholm, Sweden; Department of Neuroradiology (S.S.), Karolinska University Hospital, Stockholm, Sweden; MR Applications and Workflow, GE Healthcare (M.J.H.), Seoul, Republic of Korea; and MR Applied Science Laboratory Europe (T.S.), GE Healthcare Stockholm, Sweden.

Please address correspondence to Hye Jin Baek, MD, PhD, Department of Radiology, Gyeongsang National University School of Medicine and Gyeongsang National University Changwon Hospital, 11 Samjeongja-ro, Seongsan-gu, Changwon 51472, Republic of Korea; e-mail: sartre81@gmail.com

-- Indicates open access to non-subscribers at www.ajnr.org

Indicates article with supplemental on-line table.

Indicates article with supplemental on-line photos.

http://dx.doi.org/10.3174/ajnr.A6427 only evaluating limited sequences. ${ }^{7-15}$ Another fast technique, synthetic MR imaging, quantitatively approaches the absolute physical properties for single-scan, multiple-contrast generation with a total scan time of 4-5 minutes. ${ }^{4-6,16,17}$ Routine clinical use of synthetic MR imaging is limited because it cannot produce DWI and T2*WI and provide inferior image quality in synthetic T2-FLAIR due to partial volume effects. ${ }^{18}$ Following technical advances, some studies have focused on ultrafast MR imaging with 5 essential sequences in patients prone to motion, combining advanced imaging techniques with optimized accelerated versions of commercially available sequences, and the studies showed sufficient image quality for diagnostic use with scan times of 2 minutes 47 seconds and 4 minutes. ${ }^{19,20}$

Recently, a new 1-minute full brain, multicontrast EPI sequence (EPIMix) was introduced, ${ }^{21}$ which can shorten scan time and generate T1-FLAIR, T2WI, T2-FLAIR, DWI, apparent diffusion coefficient, and $\mathrm{T} 2{ }^{\star} \mathrm{WI}$ sequences in 78 seconds. Only 1 study showed comparable diagnostic performance between EPIMix and routine brain MR imaging for clinical use. ${ }^{22}$ However, the EPIMix studies did not fully evaluate image quality according to individual 


\begin{tabular}{|c|c|c|c|c|c|c|c|c|c|c|c|}
\hline \multirow[b]{2}{*}{ Imaging Parameter } & \multicolumn{6}{|c|}{ EPIMix MR Imaging } & \multicolumn{5}{|c|}{ Routine Brain MR Imaging } \\
\hline & & T1-FLAIR & T2WI & T2-FLAIR & DWI & $\mathrm{T} 2 * \mathrm{WI}$ & T1-FLAIR & T2WI & T2-FLAIR & DWI & SWAN \\
\hline FOV $(\mathrm{cm})$ & & 24 & 24 & 24 & 24 & 24 & 22 & 22 & 22 & 22 & 22 \\
\hline Section thickness (mm) & & 5 & 5 & 5 & 5 & 5 & 5 & 5 & 5 & 5 & 1.2 \\
\hline TR (ms) & & 1300 & 2447 & 5818 & 2447 & 2542 & 2400 & 5175 & 9000 & 5417 & 32.8 \\
\hline TI (ms) & & 570.2 & & 2751 & & & 850 & & 2465 & & \\
\hline $\mathrm{TE}(\mathrm{ms})$ & & 19.3 & 109 & 115 & 109 & 30.5 & 25.8 & 119.4 & 102.7 & 73.1 & 22.9 \\
\hline $\mathrm{ETL}$ (ms) & & & & & & & 5 & 26 & 32 & 1 & 3 \\
\hline Frequency matrix & & 180 & 180 & 180 & 180 & 180 & 360 & 512 & 288 & 128 & 288 \\
\hline Phase matrix & & 180 & 180 & 180 & 180 & 180 & 280 & 512 & 288 & 192 & 260 \\
\hline Flip angle & & $90^{\circ}$ & $90^{\circ}$ & $90^{\circ}$ & $90^{\circ}$ & $90^{\circ}$ & $90^{\circ}$ & $90^{\circ}$ & $90^{\circ}$ & $90^{\circ}$ & $15^{\circ}$ \\
\hline Bandwidth (kHz) & & 250 & 250 & 250 & 250 & 250 & 50 & 50 & 41.67 & 250 & 41.67 \\
\hline $\begin{array}{l}\text { Parallel imaging } \\
\text { acceleration factor }\end{array}$ & & & & & & & & ARC 3 & ARC 3 & ASSET 2.5 & ASSET 2.5 \\
\hline Net scan time (min:sec) & 1:12 & & & & & & 1:58 & $2: 29$ & $2: 25$ & 1:19 & $2: 33$ \\
\hline Total scan time (min:sec) & $1: 28$ & & & & & & 1:58 & $2: 56$ & $2: 49$ & 1:37 & $2: 39$ \\
\hline
\end{tabular}

Note:-ARC indicates autocalibrating reconstruction for Cartesian imaging; ASSET, array spatial sensitivity encoding technique; ETL, echo-train length.

sequences, and they acquired images using the same MR imaging scanners at the same institution. ${ }^{21,22}$ Therefore, here, we evaluated the clinical feasibility of EPIMix on a different MR imaging scanner by comparing EPIMix with the routine MR imaging protocol. We hypothesized that EPIMix would be noninferior to the routine protocol for clinical use with a 10 -fold reduction in scan time to obtain the basic 5 sequences.

\section{MATERIALS AND METHODS Patients}

We retrospectively reviewed the database of our institution and identified 146 patients who underwent diagnostic brain MR imaging examinations, including EPIMix and routine protocols with the 5 basic sequences in a single examination session from January 2019 to May 2019. The included patients were 75 men and 71 women (age range, 15-89 years; mean age, 57.2 years). MR imaging examinations were performed for headache $(32 / 146$, $21.9 \%)$, dizziness or vertigo $(29 / 146,19.9 \%)$, brain metastasis work-up $(17 / 146,11.6 \%)$, follow-up of treated intracranial aneurysm $(10 / 146,6.8 \%)$, weakness of the extremities $(7 / 146,4.8 \%)$, syncope $(7 / 146,4.8 \%)$, brain tumor follow-up $(7 / 146,4.8 \%)$, sensory change $(6 / 146,4.1 \%)$, altered mental status $(6 / 146,4.1 \%)$, parkinsonism $(5 / 146,3.4 \%)$, infarction follow-up (5/146, 3.4\%), intracranial hemorrhage $(3 / 146,2.1 \%)$, dysarthria $(2 / 146,1.4 \%)$, and other reasons (10/146, 6.8\%).

This study was approved by the institutional review board at Gyeongsang National University Changwon Hospital. The institutional review board determined that patient approval and informed consent were not required due to the retrospective nature of the study.

\section{Imaging Acquisition}

MR imaging was performed using a 3T system (Signa Architect; GE Healthcare, Milwaukee, Wisconsin) with a 48-channel head coil. Both the EPIMix and routine MR imaging protocols included 5 basic image contrasts: T1-FLAIR, T2WI, T2-FLAIR, DWI, and susceptibility-weighted angiography (SWAN)/T2*WI. At our institution, we use the ultrafast protocol for clinical purposes in specific clinical situations (such as motion-prone patients or patients with time-critical disease), and we routinely acquire images using the 2 sets of MR imaging protocols (EPIMix and routine protocols) in such clinical cases.

\section{EPIMix Brain MR Imaging Protocol}

The EPIMix pulse sequence was acquired using a Discovery $750 \mathrm{w}$ 3T or an Optima 450 1.5T MR imaging system (GE Healthcare) in the original study. ${ }^{21}$ Compared with the initial version of EPIMix, ${ }^{21}$ the present version was improved by reducing the wait time before the T2-FLAIR block, and 2 EPI echoes were applied for DWI and T2-FLAIR to increase the SNR, with the same version as that used in a recent study. ${ }^{22}$ The present version of EPIMix was optimized for our MR imaging system; therefore, EPIMix was acquired using a section thickness of $5 \mathrm{~mm}$, in-plane acceleration factor of $\mathrm{R}=3$, and section number of 28 . The EPIMix protocol is detailed in the Table. It included axial T1FLAIR, T2WI, T2-FLAIR, DWI, and T2*WI. More detailed technical aspects of EPIMix can be found in a previous study. ${ }^{21}$ The net scan time was 1 minute 12 seconds, and the total scan time was 1 minute 28 seconds.

\section{Routine Brain MR Imaging Protocol}

The routine brain MR imaging protocol included 5 basic sequences of axial T1-FLAIR, T2WI, T2-FLAIR, DWI, and SWAN. Details of routine MR imaging protocols can be found in the Table. The net scan time of routine MR imaging was 10 minutes 44 seconds, and the total scan time was 11 minutes 59 seconds.

\section{Image Analysis}

All datasets were anonymized with randomization, and 2 readers, blinded to patient information, reviewed all images using a PACS. Two attending neuroradiologists with 9 and 4 years of experience, respectively, performed an independent analysis of both brain MR imaging protocols to evaluate the image quality of EPIMix from a clinical feasibility perspective. On-line Figure 1 shows a representative example of the images obtained with the 2 different MR imaging protocols. For interpretation, each reader first analyzed and assessed all EPIMix sequences. After a 4-week memory-washout period, the readers analyzed and assessed all routine MR imaging sequences. The evaluation included 


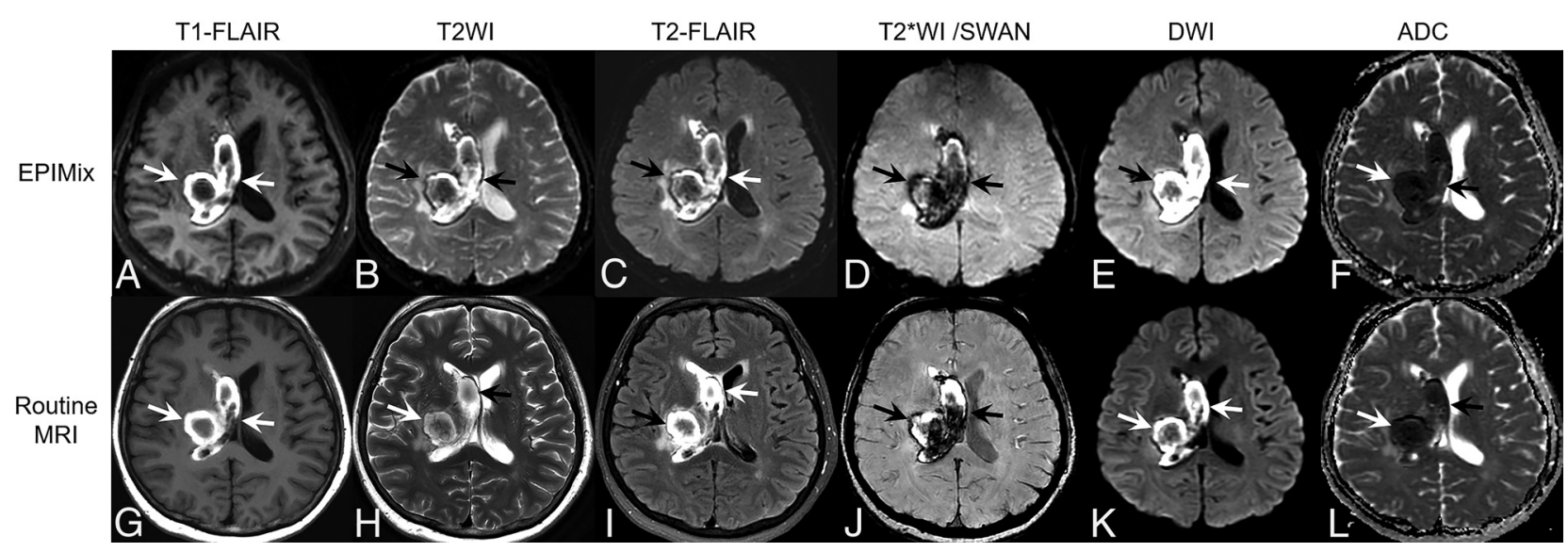

FIG 1. A 59-year-old woman with intracranial hemorrhage. A right thalamic hemorrhage with intraventricular extension to the right lateral ventricle shows mainly hyperintensity on T1-FLAIR ( $A$ and $G$ ), hyperintensity on T2WI ( $B$ and $H$ ) and T2-FLAIR ( $C$ and $I)$, blooming artifacts on T2*WI $(D)$ and SWAN ( () , and diffusion restriction on DWI ( $E$ and $K)$ (arrows in each sequence). These findings are well-visualized on both EPIMix MR imaging $(A-F)$ and routine $M R$ imaging $(G-L)$.

assessments of overall image quality, visualization of several anatomic structures, and the severity of artifacts.

For T1-FLAIR, T2WI, and T2-FLAIR, the image quality was rated according to the following 4 criteria: 1 , overall image quality; 2 , differentiation of gray matter-white matter at the level of the lateral ventricles; 3 , demarcation of the basal ganglia at the level of the foramen of Monro; and 4, demarcation of the sulci. For DWI and $\mathrm{T} 2{ }^{*} \mathrm{WI} / \mathrm{SWAN}$, the overall image quality was rated. Each criterion was graded on a Likert scale of $1-4$ points: ${ }^{23} 1$, inadequate (not acceptable for diagnostic use); 2, sufficient (acceptable for diagnostic use but with minor issues); 3 , good (acceptable for diagnostic use); and 4, excellent (acceptable for diagnostic use).

The severity of artifacts was rated for all sequences according to the following 2 criteria: 1 , motion artifacts; 2 , susceptibility artifacts. Each criterion was also scored on a 4-point Likert scale, in which ${ }^{23}$ images contain the following: 1, severe artifacts (not acceptable for diagnostic use; 2, moderate artifacts (sufficient for diagnostic use but with minor issues); 3 , mild artifacts (acceptable for diagnostic use because minor artifacts do not adversely affect diagnostic use); and 4, images do not contain visible artifacts (acceptable for diagnostic use). Then, the readers' ratings were simply dichotomized as follows: 1) image quality assessments, sufficient for clinical use ( 2 points) versus excellent for clinical use ( $\geq 3$ points); and 2) artifact assessments, severe degree (2 points) versus lesser degree ( $\geq 3$ points) because there was no case assigned 1 point in the image quality assessment.

\section{Statistical Analysis}

We assigned numeric values to the image quality assessments. Although we did not directly statistically compare the mean values of the readers' ratings because they did not strictly comprise continuous variables, we decided to present a summary of the readers' ratings for each MR imaging sequence, expressed as mean $\pm \mathrm{SD}$. We used the Wilcoxon signed rank test to compare ordinal variables and the McNemar test to compare dichotomous variables between the $2 \mathrm{MR}$ imaging protocols. We also calculated the disagreement percentage of the image quality between the proposed EPIMix protocol and the routine protocol.
Additionally, we present the interobserver agreement as the percentage agreement. We performed all statistical analyses with statistical software (SPSS, Version 24.0; IBM, Armonk, New York), and we considered $P$ values $<.05$ statistically significant.

\section{Data Availability Statement}

The anonymized data of this study will be shared on request by any qualified investigator.

\section{RESULTS \\ Study Population}

Of 146 patients, 117 (80.1\%) had abnormal MR imaging findings and $29(19.9 \%)$ had findings that were considered normal. There were the following diagnoses: ischemic/hemorrhagic stroke or small-vessel disease $(79 / 146,54.1 \%)$, intracranial neoplasm (9/ $146,6.2 \%)$, vascular abnormality $(9 / 146,6.2 \%)$, infectious or demyelinating disease $(5 / 146,3.4 \%)$, metabolic or degenerative disorder (4/146, 2.7\%), congenital CNS malformation (1/146, $0.7 \%)$, and miscellaneous, including trauma or indeterminate condition (10/146, 6.8\%).

\section{Image Quality Assessment}

The On-line Table provides the mean scores from the 2 readers, the interobserver percentage agreement of the readers' ratings, and disagreement percentages of the dichotomized mean scores for the 5 sequences of the $2 \mathrm{MR}$ imaging protocols. Although the overall image quality of all sequences derived from EPIMix, except for DWI, showed a significant difference $(P<.05)$ between the EPIMix and the routine protocols, the EPIMix sequence showed at least sufficient image quality, with an assessment rating of $>2$ points on average (Figs 1-3 and On-line Figs 1-2). Among the 5 EPIMix sequences, T2WI had the lowest mean score in the overall image quality assessment. In contrast, the overall quality of EPIMix DWI was not significantly different from that of routine DWI $(P>.05)$. EPIMix T1-FLAIR, T2WI, T2-FLAIR, and $\mathrm{T} 2{ }^{*} \mathrm{WI}$ showed significant susceptibility artifacts compared with the corresponding sequences of routine $\mathrm{MR}$ imaging $(P<.05)$. However, fewer motion artifacts tended 


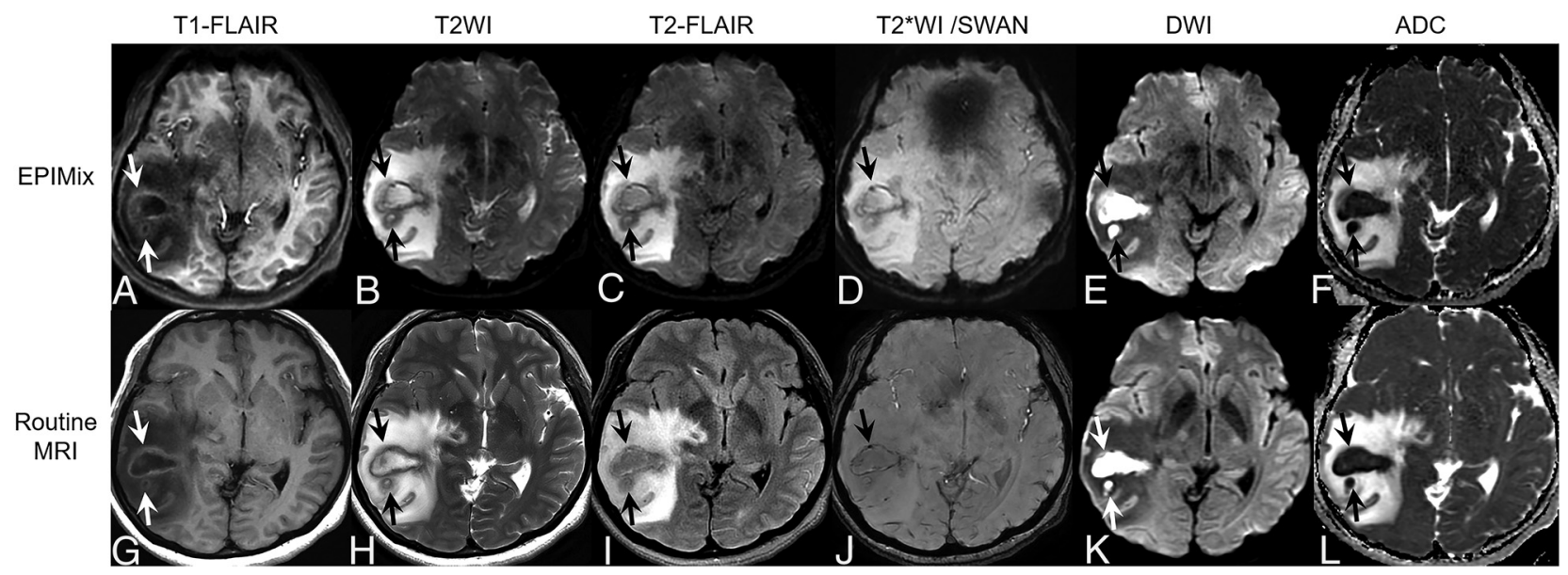

FIG 2. A 48-year-old woman with brain abscesses. There are 2 irregularly shaped masslike lesions with perilesional edema in the right temporal lobe. The internal content shows hypointensity on T1-FLAIR ( $A$ and $G)$ and hyperintensity on T2WI $(B$ and $H$ ) and T2-FLAIR (C and $I)$ (arrows). The internal content shows diffusion restriction on DWI ( $E$ and $K)$ (arrows). There are blooming artifacts on T2*WI (D) and SWAN (l) (arrows). These findings are well-visualized on both EPIMix MR imaging $(A-F)$ and routine MR imaging $(G-L)$.

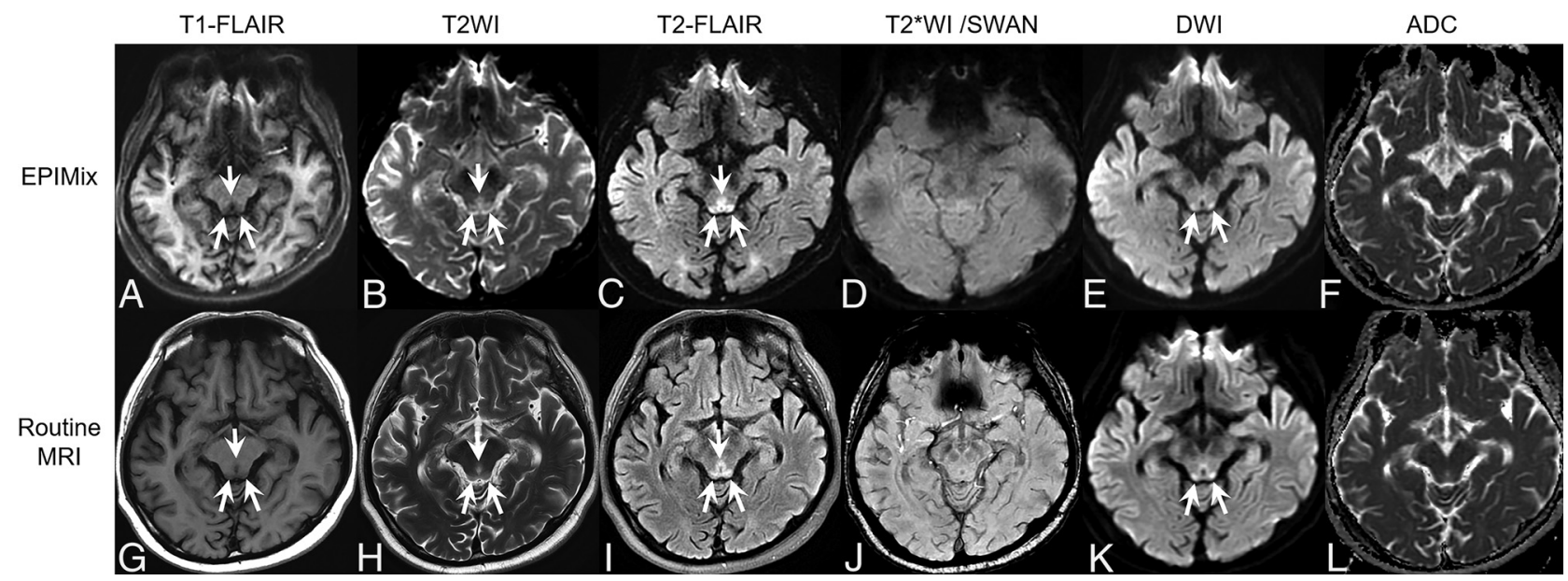

FIG 3. A 52-year-old man with Wernicke encephalopathy. Signal changes in the tectal plate of the midbrain and periaqueductal gray matter showing hypointensity on TI-FLAIR ( $A$ and $G)$, hyperintensity on T2WI $(B$ and $H)$ and T2-FLAIR $(C$ and $I)$, and hyperintensity on DWI $(E$ and $K)$ are well-visualized on both EPIMix MR imaging $(A-F)$ and routine MR imaging $(G-L)$ (arrows in each sequence).

to be observed in the EPIMix than in the routine protocol. Additionally, there was no difficulty in detecting small lesions on EPIMix in the enrolled patients (Fig 3).

\section{DISCUSSION}

In the current study, we assessed the diagnostic image quality of the EPIMix sequence outside the institution that developed it using a different scan environment and found that the EPIMix protocol had sufficient image quality with fewer motion artifacts with a short scan time of approximately 1 minute. However, the EPIMix protocol was inferior in terms of overall image quality, with more severe susceptibility artifacts than those observed in routine brain MR imaging.

Previous clinical fast brain MR imaging protocol studies have shown comparable image quality and high diagnostic concordance with conventional MR imaging. ${ }^{19,20}$ However, the scan time in those studies varied, ranging from 2 to 10 minutes, being much longer than that of the present EPIMix protocol. ${ }^{14,15,19}$ To date, the fastest brain MR imaging protocol reported required 1 minute 7 seconds of net scan time; however, the total scan time was 2 minutes 47 seconds due to the prescanning time before each sequence, ${ }^{20}$ whereas the EPIMix protocol, including all 5 essential sequences, required only a single prescan at the start of the MR imaging examination. Moreover, the EPI readout is more SNR-efficient than the corresponding fast spin-echo readout, and the smaller matrix size can accelerate the EPIMix scan. ${ }^{21}$ On the basis of these advantages, the total EPIMix scan time appears to be the shortest among those of the reported ultrafast protocols.

The EPIMix protocol uses different magnetization preparations combined with 1 of 2 sets of EPI readout trains to directly acquire the weighted contrast in a short scan time of 1 minute. ${ }^{21}$ It also uses a dynamic combination of 11 sequence modules with a unique role in the acquisition to generate a specific image contrast, and 2 EPI readout modules are reused throughout the sequence and are prepended by other modules to form the 
desired MR contrasts. ${ }^{21}$ This new 1-minute EPIMix protocol showed diagnostic performance comparable with that of routine brain MR imaging in terms of disease identification and categorization in a previous clinical study; ${ }^{22}$ however, the authors did not analyze image quality and characteristics on the basis of individual sequences derived from EPIMix. ${ }^{22}$ From the perspective of feasibility, analyses of the image quality and characteristics of each image sequence are essential to apply EPIMix to clinical use to cover a wide range of patient groups because important image sequences for diagnosis differ depending on pathology. Therefore, we performed image quality analysis for individual image sequences to evaluate the clinical feasibility of EPIMix in various brain pathologies compared with the routine brain MR imaging protocol.

In this study, EPIMix T1-FLAIR showed excellent anatomic delineation, including differentiation of gray matter-white matter, comparable with that of the routine sequence. However, geometric distortion, especially in the skull base and frontal lobe, downgraded the overall image quality of EPIMix T1-FLAIR. Although a higher acceleration factor reduces geometric distortion and $\mathrm{T} 2{ }^{*}$ blurring on images with increasingly effective image sharpness, the susceptibility artifacts of EPIMix could not be completely overcome and there remained some g-factor-related SNR loss. ${ }^{21}$ Additionally, EPIMix T2-FLAIR showed good overall image quality with good-to-excellent anatomic delineation. Our findings are consistent with those of previous studies that showed image quality and diagnostic performance comparable with that of conventional T2-FLAIR in patients with acute stroke, ${ }^{24}$ and the contrast-to-noise ratio of EPI-FLAIR was also comparable with that of conventional FLAIR. ${ }^{25}$ However, EPI T2-FLAIR may be particularly useful in only limited clinical situations in the acute setting because it involves inherently unavoidable susceptibility artifacts. On EPIMix T2WI, the overall image quality and anatomic delineation were far inferior to those of the other EPIMix sequences because T2WI is basically a $b=0$ image. Therefore, it is essential to improve the image quality of T2WI to broaden the clinical use of EPIMix. Furthermore, EPIMix has a wellknown limitation of unavoidable geometric distortion and susceptibility artifacts in all sequences, especially in the skull base, frontal lobe, posterior fossa, and brain stem. These artifacts may limit diagnostic performance in these regions and render EPIMix unsuitable for patients undergoing stereotactic surgery or radiation therapy and for postoperative followup imaging. ${ }^{21}$

Despite these shortcomings, EPIMix can generate all 5 essential sequences in 1 minute with minimal bore time for the patients. Therefore, the shorter scan time allows EPIMix to be used as a motion-resistant MR imaging protocol in certain clinical situations such as for use with patients prone to motion, pediatric or pregnant patients, or patients with claustrophobia or pain, by decreasing patient anxiety and reducing the need for sedation. It also has superior soft-tissue contrast compared with $\mathrm{CT}$ without generating radiation during the examination, and EPIMix DWI has excellent image quality, comparable with that of routine DWI. Therefore, EPIMix can be a useful option in patients with time-critical diseases.
Furthermore, if EPIMix is run at the beginning of an examination, it may also help in the planning of detailed conventional brain MR imaging sequences.

This study has several limitations. First, it was retrospective and may have, therefore, involved unavoidable selection bias. Second, we did not perform quantitative analysis such as of the contrast-tonoise ratio or SNR because the shorter scan time inevitably leads to degraded overall image quality as also reported by previous studies. ${ }^{15,19,20}$ Although the image quality analysis is limited by the reader's subjective judgment, the readers could intuitively perceive the effect of the contrast-to-noise ratio and SNR in the 2 brain MR imaging protocols during the image quality analysis. Third, we could not randomize the acquisition order of EPIMix and routine MR imaging because of the retrospective study design. Fourth, we did not perform a direct comparison between the EPIMix protocol and brain CT in this study. Further comparative studies with head CT would be helpful to confirm the clinical utility of our EPIMix protocol as a proper CT substitute. However, further studies need to be carefully designed because CT involves radiation exposure. Fifth, interobserver agreement between the 2 readers was provided as a percentage agreement because $\kappa$ statistics provided a paradoxically low value due to an imbalance in the number of concordant and discordant pairs. ${ }^{26,27}$ Last, EPIMix is a noncommercialized sequence under technical development. It is, therefore, challenging to immediately apply it on different MR imaging scanners from multiple vendors.

\section{CONCLUSIONS}

The overall EPIMix image quality was sufficient for diagnostic use with fewer motion artifacts. However, EPIMix is limited by its inherent geometric distortion, being particularly problematic in the skull base near the tissue-air boundaries, and has lower resolution compared with fast spin-echo-based series in a routine protocol. Therefore, it cannot completely replace the routine MR imaging protocol at this time; however, it may be a feasible option in specific clinical situations such as screening, time-critical disease, or patients prone to motion. After further development of the improved EPIMix version, future studies with a larger sample size will help expand the clinical indications of EPIMix and validate our results.

Disclosures: Tim Sprenger-UNRELATED: Employment: GE Healthcare, Comments: Tim Sprenger is one of the joint developers of the multicontrast EPI sequences with Professor Skare, and they provided the multicontrast EPI research pulse sequence for this study. However, GE Healthcare had no role in the design of the study, data analyses, or data interpretation.

\section{REFERENCES}

1. Heidemann RM, Ozsarlak O, Parizel PM, et al. A brief review of parallel magnetic resonance imaging. Eur Radiol 2003;13:2323-37 CrossRef Medline

2. Deshmane A, Gulani V, Griswold MA, et al. Parallel MR imaging. J Magn Reson Imaging 2012;36:55-72 CrossRef Medline

3. Barth M, Breuer F, Koopmans PJ, et al. Simultaneous multislice (SMS) imaging techniques. Magn Reson Med 2016;75:63-81 CrossRef Medline

4. Warntjes JB, Leinhard OD, West J, et al. Rapid magnetic resonance quantification on the brain: Optimization for clinical usage. Magn Reson Med 2008;60:320-29 CrossRef Medline

5. Gulani V, Schmitt P, Griswold MA, et al. Towards a single-sequence neurologic magnetic resonance imaging examination: multiple- 
contrast images from an IR TrueFISP experiment. Invest Radiol 2004;39:767-74 CrossRef Medline

6. Hagiwara A, Warntjes M, Hori M, et al. SyMRI of the brain: rapid quantification of relaxation rates and proton density, with synthetic MRI, automatic brain segmentation, and myelin measurement. Invest Radiol 2017;52:647-57 CrossRef Medline

7. Ashley WW Jr, McKinstry RC, Leonard JR, et al. Use of rapid-sequence magnetic resonance imaging for evaluation of hydrocephalus in children. J Neurosurg 2005;103:124-30 CrossRef Medline

8. Niederhauser BD, McDonald RJ, Eckel LJ, et al. Retrospective review of rapid pediatric brain MR imaging at an academic institution including practice trends and factors affecting scan times. AJNR Am J Neuroradiol 2013;34:1836-40 CrossRef Medline

9. Ryan ME, Jaju A, Ciolino JD, et al. Rapid MRI evaluation of acute intracranial hemorrhage in pediatric head trauma. Neuroradiology 2016;58:793-99 CrossRef Medline

10. Wagner MW, Kontzialis M, Seeburg D, et al. Acute brain imaging in children: can MRI replace CT as a screening tool? J Neuroimaging 2016;26:68-74 CrossRef Medline

11. Kralik SF, Yasrebi M, Supakul N, et al. Diagnostic performance of ultrafast brain MRI for evaluation of abusive head trauma. AJNR Am J Neuroradiol 2017;38:807-13 CrossRef Medline

12. Missios S, Quebada PB, Forero JA, et al. Quick-brain magnetic resonance imaging for nonhydrocephalus indications. J Neurosurg Pediatr 2008;2:438-44 CrossRef Medline

13. Penzkofer AK, Pfluger T, Pochmann Y, et al. MR imaging of the brain in pediatric patients: diagnostic value of HASTE sequences. AJR Am J Roentgenol 2002;179:509-14 CrossRef Medline

14. U-King-Im JM, Trivedi RA, Graves MJ, et al. Utility of an ultrafast magnetic resonance imaging protocol in recent and semi-recent strokes. J Neurol Neurosurg Psychiatry 2005;76:1002-05 CrossRef Medline

15. Nael K, Khan R, Choudhary G, et al. Six-minute magnetic resonance imaging protocol for evaluation of acute ischemic stroke: pushing the boundaries. Stroke 2014;45:1985-91 CrossRef Medline

16. Tanenbaum LN, Tsiouris AJ, Johnson AN, et al. Synthetic MRI for clinical neuroimaging: results of the Magnetic Resonance Image
Compilation (MAGiC) prospective, multicenter, multireader trial. AJNR Am J Neuroradiol 2017;38:1103-10 CrossRef Medline

17. Ryu KH, Baek HJ, Moon JI, et al. Initial clinical experience of synthetic MRI as a routine neuroimaging protocol in daily practice: a single-center study. J Neuroradiol 2019 Apr 2. [Epub ahead of print] CrossRef Medline

18. Vargas MI, Boto J, Delatre BM. Synthetic MR imaging sequence in daily clinical practice. AJNR Am J Neuroradiol 2016;37:E68-E69 CrossRef Medline

19. Prakkamakul S, Witzel T, Huang S, et al. Ultrafast brain MRI: clinical deployment and comparison to conventional brain MRI at 3T. J Neuroimaging 2016;26:503-10 CrossRef Medline

20. Ryu KH, Choi DS, Baek HJ, et al. Clinical feasibility of 1-min ultrafast brain MRI compared with routine brain MRI using synthetic MRI: a single center pilot study. J Neurol 2019;266:431-39 CrossRef Medline

21. Skare S, Sprenger T, Norbeck O, et al. A 1-minute full brain MR exam using a multicontrast EPI sequence. Magn Reson Med 2018;79:3045-54 CrossRef Medline

22. Delgado AF, Kits A, Bystam J, et al. Diagnostic performance of a new multicontrast one-minute full brain exam (EPIMix) in neuroradiology: a prospective study. J Magn Reson Imaging 2019;50:182433 CrossRef Medline

23. Likert R. A technique for the measurement of attitudes. Archives of Psychology 1932;22:1-55 https://psycnet.apa.org/record/1933-01885001. Accessed July 28, 2019

24. Meshksar A, Villablanca JP, Khan R, et al. Role of EPI-FLAIR in patients with acute stroke: a comparative analysis with FLAIR. AJNR Am J Neuroradiol 2014;35:878-83 CrossRef Medline

25. Korogi Y, Sugahara T, Shigematsu Y, et al. Ultrafast FLAIR imaging with single-shot echo-planar technique in evaluation of intracranial lesions. Comput Med Imaging Graph 1999;23:119-26 CrossRef Medline

26. Viera AJ, Garrett JM. Understanding interobserver agreement: the kappa statistic. Fam Med 2005;37:360-63 Medline

27. Feinstein AR, Cicchetti DV. High agreement but low kappa, I: the problems of two paradoxes. J Clin Epidemiol 1990;43:543-49 CrossRef Medline 